\title{
Environment, epigenetics and neurodegeneration: Focus on nutrition in Alzheimer's disease
}

\author{
Vincenzina Nicolia a , Marco Lucarelli b,c ${ }^{\text {, }}$ Andrea Fuso ${ }^{\mathrm{d}, \mathrm{e}, *}$ \\ a Dept. of Surgery “P. Valdoni”, Via A. Scarpa 16, 00161, Sapienza University of Rome, Italy \\ ${ }^{\mathrm{b}}$ Dept. of Cellular Biotechnologies and Hematology, Viale del Policlinico 155, 00161, Sapienza University of Rome, Italy \\ c Pasteur Institute Cenci Bolognetti Foundation, Viale Regina Elena 291, 00161, Sapienza University of Rome, Italy \\ d Dept. of Psychology, Via dei Marsi, 78 00183, Sapienza University of Rome, Italy \\ e European Center for Brain Research (CERC)/IRCCS Santa Lucia Foundation, Via del Fosso di Fiorano 64-65, 00143 Rome, Italy
}

\section{A R T I C L E I N F O}

\section{Article history:}

Received 1 October 2014

Received in revised form 7 October 2014

Accepted 10 October 2014

Available online 14 October 2014

Section Editor: Kurt Borg

Keywords:

Epigenetics

DNA methylation

Neurodegeneration

Alzheimer's Disease

\begin{abstract}
A B S T R A C T
Many different environmental factors (nutrients, pollutants, chemicals, physical activity, lifestyle, physical and mental stress) can modulate epigenetic markers in the developing and adult organism. Epigenetics, in turn, can cause and is associated with several neurodegenerative and aging-dependent human diseases. Alzheimer's disease certainly represents one of the most relevant neurodegenerative disorders due to its incidence and its huge socio-economic impact. Therefore, it is easy to understand why recent literature focuses on the epigenetic modifications associated with Alzheimer's disease and other neurodegenerative disorders.

One of the most intriguing and, at the same time, worrying evidence is that even "mild" environmental factors (such as behavioral or physical stress) as well as the under-threshold exposure to pollutants and chemicals, can be effective. Finally, even mild nutrients disequilibria can result in long-lasting and functional alterations of many epigenetic markers, although they don't have an immediate acute effect. Therefore, we will probably have to re-define the current risk threshold for many factors, molecules and stresses.

Among the many different environmental factors affecting the epigenome, nutrition represents one of the most investigated fields; the reasons are probably that each person interacts with nutrients and that, in turn, nutrients can modulate at molecular level the epigenetic biochemical pathways. The role that nutrition can exert in modulating epigenetic modifications in Alzheimer's disease will be discussed with particular emphasis on the role of $B$ vitamins and DNA methylation.
\end{abstract}

(c) 2014 Elsevier Inc. All rights reserved.

\section{Introduction}

The term "epigenetics" refers to different biological traits that are responsible for regulation of gene expression through the interaction with the DNA sequence of a gene. Although many different phenomena have been included in the epigenetic traits, and no clear definition of which is epigenetic and which is not exists, it is usually agreed that the three most relevant epigenetic modifications are DNA methylation, histone modifications and miRNAs. Histone modifications (acetylation, methylation, phosphorylation, ubiquitination) and DNA methylation, together, constitute a higher-level epigenetic modification defined as chromatin remodeling.

Epigenetic modifications modulate gene expression during several physiological processes, mainly during embryonic development and cellular differentiation, although we have been observing an increasing

\footnotetext{
* Corresponding author at: Dept. of Psychology, Via dei Marsi, 78, 00183 Rome, Italy. E-mail address: andrea.fuso@uniroma1.it (A. Fuso).
}

number of pathological processes in which epigenetic mechanisms appear to be involved or even causative (Portela and Esteller, 2010).

\section{Epigenetics and neurodegeneration}

The first evidences that epigenetic mechanisms, DNA methylation in particular, were associated with specific diseases have been given by studies on tumors (Jones and Buckley, 1990) and on three neurological diseases: Fragile X, ICF and Rett syndromes (Fuso, 2013). More recently, several studies have stressed the relationship between epigenetic alterations and neurodegenerative disorders (Marques et al., 2011). As a matter of fact, many diseases have multifactorial origin, depending not only on genetic but also on stochastic and environmental factors. These diseases are defined "complex disorders" and include, besides neurodegenerative diseases, cardiovascular disease, cancer, diabetes and neuropsychiatric diseases. Alzheimer's disease (AD) is the most common cause of dementia and the most expensive chronic disease, constituting a huge social and economical problem. AD is a clinical syndrome characterized by memory impairment in the short and long term 
of at least one of the primary mental activities. Its spread, the lack of effective therapies and the huge costs indicate $\mathrm{AD}$ as one of the highest social impacts (Zhu and Sano, 2006). Approximately 33.9 million people worldwide have $\mathrm{AD}$ and prevalence will increase 3-4 times in the next 40 years.

AD presents as familial and sporadic forms and although different polymorphisms and risk factors have been associated with onset and progression of the disease, the precise way by which the environment contributes to neurodegeneration is still unclear. Increasing evidences suggest that environmental factors may contribute to neurodegeneration through the modulation of the epigenetic pattern resulting in alterations of gene expression programs. Epigenetics refers to any process that alters gene activity without changing the primary DNA sequence and leads to modifications that can be transmitted to daughter cells. It is a relatively novel area of research that is currently attracting a high level of interest. During the lifespan of complex organisms, epigenetic changes can occur as early as embryonic developmental processes and constitute a fundamental way to regulate the very complex and coordinated gene expression pattern. The idea that epigenetic modifications are also associated with the aging processes and with the loss of phenotypic plasticity is more recent. Since aging is the most important risk factor for sporadic AD, we can infer that deviations from the "healthy" epigenetic patterns accumulate with aging and in neurodegeneration, contributing to the etiology of AD and other neurodegenerative disorders (Fuso, 2013).

DNA methylation, which consists in the binding of a methyl group $\left(-\mathrm{CH}_{3}\right)$ to the carbon in position 5 of a cytosine moiety, appears to be frequently involved in the processes associated both with healthy and diseased aging, particularly in neurological and neurodegenerative diseases.

The first example has been the fragile X syndrome (Jin and Warren, 2000): a mental retardation syndrome caused by a CGG triplet repeats expansion in the $5^{\prime}$-untranslated region of the fragile $\mathrm{X}$ mental retardation-1 (FMR1) gene. The CGG repeats result hypermethylated in affected subjects causing FMR1 gene repression. Also ICF (Immunodeficiency, Centromere instability and Facial abnormalities) and Rett syndromes were originally related to methylation since they are characterized by mutations in genes encoding for proteins involved in the regulation of the DNA methylation homeostasis (Kumar, 2000). Today we know that DNA methylation alterations can contribute to a large number of different neurological and neurodegenerative diseases as a result of different mechanisms. Many of the diseases associated with aberrant DNA methylation show methylation-based imprinting alterations; these include Angelman syndrome, Silver-Russell syndrome, Prader-Willi syndrome and Beckwith-Wiedemann syndrome, schizophrenia and autism (Falls et al., 1999).

In the recent years, it was possible to demonstrate that genomic and sequence-specific DNA methylation patterns dynamically changes during the lifespan and are characteristic and distinct between different brain areas. It has also been demonstrated that epigenetic changes can contribute to $\mathrm{AD}$ onset and course and that specific loci, such as PSEN1, are demethylated in AD subjects (reviewed in Mastroeni et al., 2011). In our laboratory, we were able at demonstrating that PSEN1 expression can be modulated by modifying the methylation homeostasis, with consequent alteration of the amyloidogenic pathway, senile plaques burden and specific cognitive tasks in AD mice as discussed in detail in the next section (Fuso, 2013; Fuso et al., 2012). Furthermore, two independent laboratories recently disclosed new specific loci with differential methylation associated with AD (De Jager et al., 2014; Lunnon et al., 2014).

All the data collected thus so far about DNA methylation in AD point out a central role for this epigenetic modification in the connection between amyloidogenic, fibrillogenic, inflammatory and oxidative pathways. All this evidence proves that DNA methylation is involved in AD-related molecular mechanisms, either as a primary cause or as a consequence of the disease; epigenetic studies at genomic and at gene-specific levels will be necessary to further disclose relevant knowledge on the epigenetic role in $\mathrm{AD}$.

\section{Environment and epigenetics}

Environmental factors are increasingly claimed to be responsible for neurodegeneration-related modification; a link between environmental induced epigenetic modification, oxidation, and repair of AD-related genes, is discussed in depth in a recent review (Coppedè and Migliore, 2010). The evidence we have been acquiring on the relationship between environment and epigenetics teaches us that many epigenetic mechanisms are potentially triggered by a plethora of environmental factors. This observation can also be seen under a slightly different perspective indicating that the modulation of the epigenetic mechanisms actually acts as a mediator of environmental stimuli. The term "environment" include, in this case, many external different processes and conditions that come in contact with the organism. These environmental factors include, among others: stressors (physical and behavioral), nutritional factors, pollutants, pesticides, chemicals, metals, physical exercise, mental exercise and lifestyle. All these factors can exert on the organism different biological effects that could determine or contribute to the onset and progression of many diseases.

Environmental factors can induce epigenetic changes in the individual in each period of the life, from the in utero period to the elderly. However, it appears obvious that these factors are becoming increasingly relevant with respect to the shift from the healthy to the diseased statuses of an individual with aging, due to the increased likelihood to encounter environmental insults or to accumulate their effects during aging. This observation also stresses the relationship between the environment-related epigenetic changes and the increasing incidence of $\mathrm{AD}$ and other neurodegenerative diseases observed in the last few decades: the longer the lifespan of an individual, the higher the possibility to receive epigenetic "hits" leading to diseased aging (Fig. 1).

On the other hand, increasing epidemiological evidence suggests that maternal environmental exposure and stimuli result in epigenetic changes occurring early in development and playing an important role in susceptibility to disease in later life, even if initially silent. Thus, epigenetic modifications provide a potential link between the environment encountered during critical periods in development (periconceptional period, gestation, and early postnatal period) and changes in gene expression that may lead to disease phenotypes (Jang and Serra, 2014).

It is of extreme interest to notice that even apparently mild environmental factors like behavioral stress or physical exercise (that do not involve the physical contact of the individual with any chemical species), could result in a long lasting alteration of epigenetic markers leading to functional phenotypic alterations. This observation should lead us to reconsider the cutoff normally considered as "risky" for many other "classical" environmental factors, such as the exposure to chemicals, pollutants, metals and pesticides. As a matter of fact, epigenetic studies reveal that exposures to these agents that are normally considered "under-threshold" could actually induce epigenetic changes.

The epigenetic effect of behavioral stress was demonstrated by studies showing that perinatal behavioral stresses in rats were responsible for altered AVP or BDNF expression, mediated by DNA methylation (Roth and Sweatt, 2011).

Physical exercise could also modify the epigenetic pathways leading to healthy or diseased aging, possibly interacting with behavioral stressors. Physical exercise improves the efficiency of the capillary system and increases the oxygen supply to the brain, thus enhancing metabolic activity and oxygen intake in neurons, and increases neurotrophin levels and resistance to stress. Regular exercise and an active lifestyle during adulthood have been associated with reduced risk and protective effects for mild cognitive impairment and AD. Similarly, studies in animal models show that moderate physical activity has positive physiological and cognitive effects that correlate with changes in transcriptional profiles possibly mediated by epigenetic modifications (Kaliman et al., 2011). 


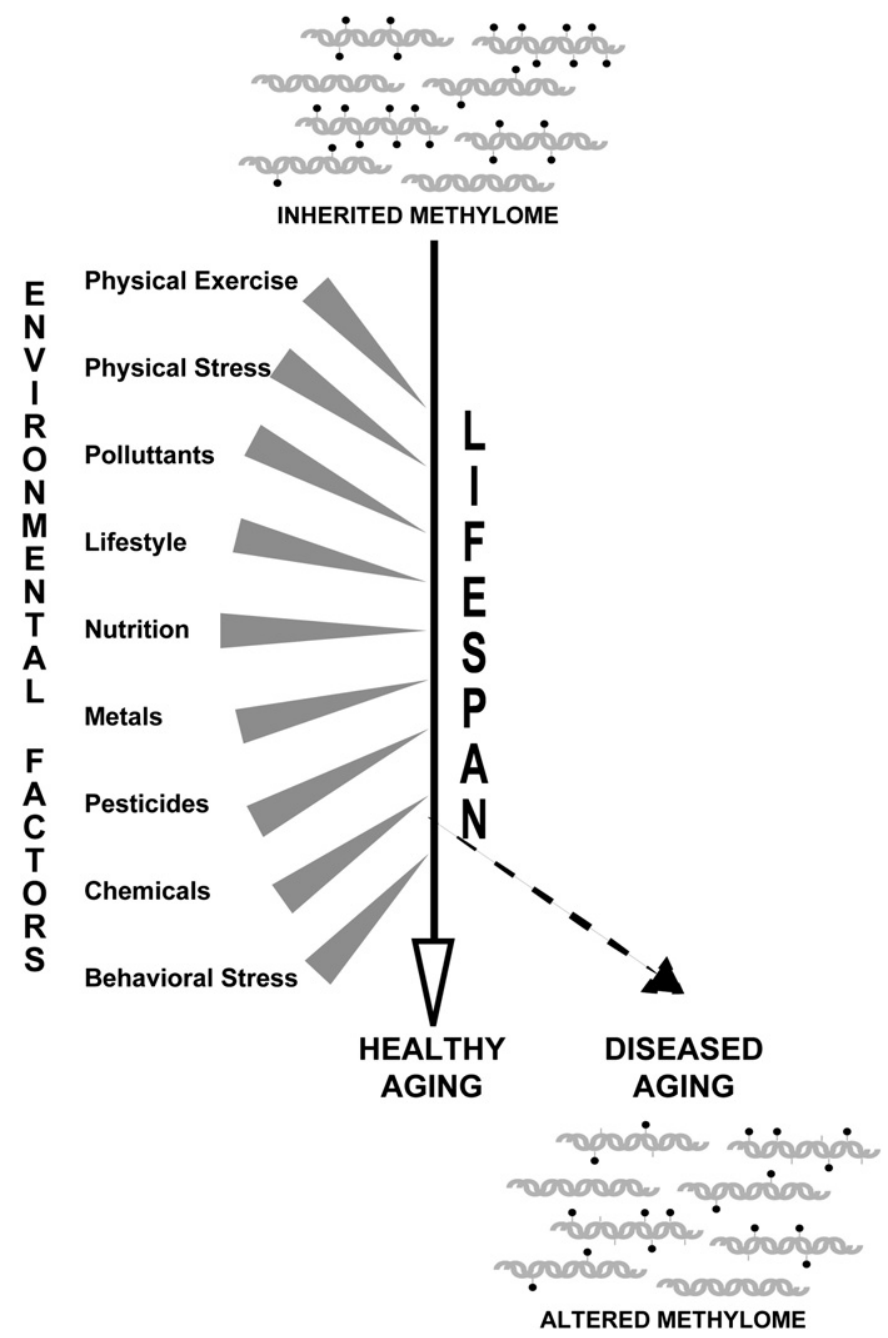

Fig. 1. Environmental factors modify aging through epigenetics. Several environmental factors can affect the metabolism of an individual by inducing epigenetic modifications. The figure depicts the inherited DNA methylation pattern which can be modified at local and sequence-specific levels by different (even mild) stressors. The modification of the inherited methylome (or of the epigenome, in general) can be responsible for the alteration of the expression of specific (aging-associated) genes, leading to conditions of diseased aging.

Epidemiological studies have successfully identified several environmental causes of disease, but these studies are often limited by methodological problems (e.g. lack of sensitivity and specificity in exposure assessment, confounding association of different environmental factors). Proposed approaches to improve observational studies of environmental associations are Mendelian randomization and the meetin-the-middle (MITM) approach (van Veldhoven et al., 2014).

Both genetic and epigenetic responses of organisms to environmental factors, including chemical exposures, influence adaptation, susceptibility to toxicity and biodiversity. In model organisms, it has been established that epigenetic alterations, including changes in DNA methylation patterns, can create a memory of the received signal. This has been shown by the analysis of epigenetic differences that develop between identical twins, discordant for the occurrence of age-related diseases. Monozygotic twin siblings share the same genotype because they are derived from the same zygote. Despite the appearance, they frequently present phenotypic differences, including their susceptibility to disease. Studies suggest that aging-associated discordance between monozygotic twins is partly related to epigenetic differences and factors changing over their lifetime (Mastroeni et al., 2011). The epigenetic drift occurring during the development probably results from a combination of stochastic and environmental factors. One example is represented by a study on twins discordant for Lewy Body Dementia that allowed postulating that epigenetic factors could play a role in Lewy Body pathology whereas in another work taking advantage of a rare set of monozygotic twins discordant for $\mathrm{AD}$, it was possible to observe significantly reduced levels of DNA methylation in temporal neocortex neuronal nuclei of the AD twins (Mastroeni et al., 2011). This result stressed the hypothesis that the effects of life events on AD risk could be mediated by epigenetic mechanisms, providing a more general potential explanation for AD discordance despite genetic similarities.

We can conclude that aging is a process characterized by genetic and epigenetic interactions, where epigenetics has an important function in determining phenotypic differences. Epigenetics also plays a key role in the development of diseases associated with aging and helps to explain the relationship between individual genetic background, environment, aging, and disease.

The characterization of the epigenetic patterns of an organism and the study of the changes occurring in this pattern when the organism undergoes environmental stimuli could bring to the identification of specific environmental factors encountered during the lifetime. It is reasonable to consider that, in the future, deviation from the "normal" epigenetic pattern and the ability at modulating epigenetic markers also at low concentrations should be taken into account when assessing environmental toxicity and/or safety of specific factors.

Epigenetics may represent a mechanistic link between environmental exposures, or genetics, and many common diseases, or may simply provide a quantitative biomarker for exposure or disease for areas of epidemiology currently lacking such measures (Bakulski and Fallin, 2014). This potential is in part reduced by issues related to the many biases challenged by the complexity and the interconnection characteristics of studies dealing with environmental factors. It is actually very difficult to separate and analyze the effect of single environmental agents; study design, measurement tools, statistical methods, and biological interpretation must be carefully considered and, hopefully, standardized. As a matter of fact, the lack of confidence with the studies, performed so far, related to the epigenetic effects of environmental factors, is due to the lack of standardized measurements, difficult in-lab reproduction of in-environment conditions and the presence of confounding interactions between several environmental factors.

\section{Focus on nutrition}

Great progress has been made in recent years to understand various facets of epigenetics, including the molecular mechanisms of epigenetic regulation of gene expression.

Nutrition and diet maybe are the main environmental factors influencing epigenetics and aging and there is growing evidence that epigenetic mechanisms may mediate the effects of nutrients, micronutrients and even non-nutrient dietary factors, pointing to them as possible causes for the development of complex diseases (McKay and Mathers, 2011). Many nutrients have shown possible epigenetic effects. However, due to the lack of consistent and reliable data on many of these factors, the present discussion will be focused on the four classes of nutrients for which we have, so far, the most consistent evidences: polyunsaturated fatty acids, $B$ vitamins and alcohol as well as metal ions and pesticides that can be consumed with the diet due to the environmental pollution.

The involvement of nutrition, environmental contaminants and heavy metals in the development of $\mathrm{AD}$ is an emerging hypothesis in the past few years (Bonda et al., 2011; Olde Rikkert et al., 2014). AD patients are frequently reported to have lower plasma levels of certain nutrients and abnormal metabolism of metal ions in the brain, compared to the healthy controls. Lower concentrations of polyunsaturated fatty acids ( $n-6$ and n-3 polyunsaturated fatty acids) and antioxidants were also found in mild AD patients (Olde Rikkert et al., 2014). Because of their high content in polyunsaturated fatty acids, neuronal membranes represent a potential target of the damaging effects of oxidative stress. The decrease of antioxidants levels observed in AD patients could result 
in major peroxidation of the lipid precursors and the derivation of membrane components; the alteration of membrane composition has been suggested to enhance the APP amyloidogenic processing and increase $A \beta$ production and aggregation in vitro (Grimm et al., 2012).

Different environmental factors (vitamin B deficiency, alcohol, pesticides and metals), associated with $\mathrm{AD}$ risk, appear able to induce epigenetic changes in the brain. Among the various mechanisms by which environmental factors could affect the progress of senescence, two pathways involve DNA methylation: the first is referred to elements able to modify DNMT activity (arsenic, cadmium, lead and pesticides), whereas the second involves the supply of metabolites of the S-adenosylmethionine cycle.

It was established that the adventitious uptake and accumulation of some metal via nutrient transporters could lead to epigenetic modification. Lead $(\mathrm{Pb})$ has been proven to exert neurotoxic effects through different mechanisms; in the last years it was shown that $\mathrm{Pb}$ could alter the expression of several DNA methyltransferases. Reduction of DNMT1 expression (the maintenance methyltransferase that copies the preexisting methylation markers onto the new strand during DNA replication) was observed in the undifferentiated PC12 cells treated with high dosage of $\mathrm{Pb}$; this alteration is able to modulate DNA methylation patterns and could cause permanent molecular damage to DNA ( $\mathrm{Li}$ et al., 2012). Moreover, Pb could inhibit DNMT3a (a de novo methyltransferase that introduces new methylation markers onto DNA) transcription by binding to its promoter (Tsai et al., in press) leading to the reduction of cytosine methylation in $\mathrm{CpG}$ islands within gene promoters. Therefore, an important consequence of $\mathrm{Pb}$ exposure could be the hypomethylation of $\mathrm{CpG}$ dinucleotide in the APP promoter leading to abnormal APP processing (Li et al., 2012).

Another heavy metal that affects DNMT activity is the cadmium (Cd). Short exposure to Cd reduces DNMT activity resulting in DNA hypomethylation. In contrast, chronic Cd exposures result in a dosedependent increase in cellular DNMT activity and concomitant DNA hypermethylation (Takiguchi et al., 2003). However, the differential effect on DNMT activity observed under short and long exposures to $\mathrm{Cd}$ is not well understood. In addition, upregulation of DNMT1 and DNMT3A/ $B$ expression has been correlated to global DNA hypermethylation in cells chronically exposed to Cd (Jiang et al., 2008). Overall, the data suggest that short exposure to $\mathrm{Cd}$ is associated with DNA hypomethylation, whereas chronic exposure is associated with DNA hypermethylation.

The intake of arsenic (As) causes the response of a variety of detoxification pathways. Methylation of inorganic As produces different organic forms that are less toxic than inorganic As (Ye et al., 2012). In mammals, cellular inorganic As is methylated by the arsenic methyltransferase (AS3MT) that utilizes S-adenosylmethionine (SAM) as the methyl donor (Gebel, 2002). Arsenic is extensively methylated during its metabolism and for this reason it has been proposed that As intake may deplete the methyl-donor pool of the cell, leading to DNA hypomethylation.

While acute toxic effects of pesticides are well known, uncertainties still remain regarding sub-acute, chronic and long-term effects. For some pesticides, like dichlorodiphenyltrichloroethane (DDT) or environmental endocrine disruptors (EDs), it is ascertained that they can cause epigenetic perturbations (Shutoh et al., 2009). Pesticides could act through several epigenetic mechanisms, including DNA methylation, histone modifications, and microRNA (miRNA) expression alteration (Baccarelli and Bollati, 2009). Further studies are warranted to evaluate if epigenetic modifications may have the role of a causal link between pesticide exposure and health effects, or rather be a sensitive marker of exposure.

Other metabolites that could impact disease states by binding to transcription factors and/or modifying chromatin structure, thereby altering gene expression patterns, are those generated during ethanol metabolism. It was shown that chronic alcohol consumption leads to either substantial DNA hypomethylation as a result of significant reduction in tissue SAM levels or histone modification. In fact, alcohol consumption is able to perturb SAM metabolism and induce degradation of DNMTs (Chen et al., 2013; Mukhopadhyay et al., 2013).

The group B vitamins (folate, B6 and B12) play important roles in SAM metabolism. After methyl group transfer, SAM is converted in S-adenosylhomocysteine (SAH), which is a strong competitive inhibitor of methyltransferases (Lu, 2000). Then, SAH is hydrolyzed to homocysteine (HCY) and adenosine by the enzyme S-adenosylhomocysteine hydrolase (SAHH); this reaction proceeds in the hydrolytic direction only if HCY and adenosine are efficiently removed. HCY can be, in turn, converted into either glutathione (GSH), an important antioxidant, via series of intermediate steps requiring vitamin $\mathrm{B} 6$ as a cofactor, or methionine. The re-methylation of $\mathrm{HCY}$ occurs through the transfer of a methyl group from 5-methyltetrahydrofolate by methionine synthase, that utilizes folate and vitamin B12 as cofactors. Deficiency of these vitamins hampers the conversion of $\mathrm{HCY}$ to methionine or cystathionine; therefore, $\mathrm{HCY}$ reverts to SAH resulting in altered methylation metabolism (Fuso et al., 2005). In the last few years, it has been demonstrated that there is a correlation between B vitamin deficiency and DNA methylation. In our laboratory we demonstrated that a transgenic mouse model of AD, the TgCRND8 mice, treated with a diet deficient of vitamin $\mathrm{B} 12, \mathrm{~B} 6$ and folate showed a decrease of SAM/SAH ratio leading to impaired methylation potential, DNMTs inhibition and DNA demethylase stimulation, PSEN1 promoter hypomethylation, PSEN1 overexpression, increased amyloid processing and deposition in senile plaques and, finally, cognitive impairment. The supplementation with SAM was able to restore control-like conditions in $\mathrm{AD}$ mice or even to partially revert the Alzheimer-like phenotype (Fuso et al., 2012). Other laboratories have confirmed the role of B vitamins and DNA methylation in molecular mechanisms related to AD onset and progression (Mastroeni et al., 2011). An alternative approach adopted in other laboratories was to control the hyperhomocysteinemia with B vitamin supplementation. Results from observational and intervention studies showed either increased risk of cognitive decline associated with higher levels of folate intake (Morris et al., 2005) or null or inconclusive results on cognitive decline (Wald et al., 2010). The reason for these inconclusive results could be due to the length of follow-up (median follow-up time $<1$ year), which may not provide the time needed for vitamin $\mathrm{B}$ supplementation to exert a protective effect. On the contrary, a randomized controlled study on elderly subjects with mild cognitive impairment showed that B-vitamin treatment was able to reduce the cerebral atrophy in gray matter regions specifically vulnerable to the $\mathrm{AD}$ process, including the medial temporal lobe (Douaud et al., 2013).

\section{Conclusions}

The concept that many different environmental stimuli can interact with the genome of an organism not just at genomic level (inducing mutations) but also at epigenetic level and that this interaction can lead to a diseased status is finally widely accepted. Nevertheless, the environment-epigenetic-disease connection still deserves more intense and more rigorous researches in order to achieve clear data and to be able to apply this knowledge to the prevention and the therapy of related diseases. One important aspect, in this sense, is represented by the availability of "epigenetic drugs" (in some case already in use in the clinical practice) and by the specific characteristic of the epigenetic modification to be (potentially) reversible.

The neurodegenerative diseases represent one of the most clear, relevant and studied examples of the environmental-associated and epigenetic-related causes of the disease; the primary reason seems to be ascribable to the increasing length of the lifespan that favors multiple environmental "insults". On the other hand, the slow development of these diseases, together with the difficulties in studying the human brain, constitutes a major drawback for the neuroepigeneticists. Another major problem is represented by the difficulty in discriminating between the effect of concurrent and interacting environmental factors of different natures on the human health. Finally, a third major hindrance 
resides on the "silent" period often existing between the environmental insult and the manifestation of the "unhealthy" effects on the organism, together with our nescience of the effective dosage at which the environmental factors exert these effects. Nonetheless, a major value of these kinds of studies will be the definition of the exact detrimental dosage of certain environmental factors for which only the toxic acute exposure is known so far.

The characterization of experimental models and the application of unbiased epigenomic approaches will be warranted to accomplish the challenging study of the epigenetic alterations induced by environmental factors.

\section{Conflict of interest}

None of the authors have competing interests or other interests that might be perceived to influence the results and/or discussion reported in this article.

\section{Acknowledgments}

This paper was supported by funding from the EC 7th Framework Program (FP7/2007-2013) grant no. 278486 "DEVELAGE” and from the Sapienza Università di Roma, Scientific Research Programs 2012 and 2013

\section{References}

Baccarelli, A., Bollati, V., 2009. Epigenetics and environmental chemicals. Curr. Opin. Pediatr. 21, 243-251.

Bakulski, K.M., Fallin, M.D., 2014. Epigenetic epidemiology: promises for public health research. Environ. Mol. Mutagen. 55, 171-183.

Bonda, D.J., Lee, H.G., Blair, J.A., Zhu, X., Perry, G., Smith, M.A., 2011. Role of methal dyshomeostasis in Alzheimer disease. Metallomics 3, 267-270.

Chen, Y., Ozturk, N.C., Zhou, F.C., 2013. DNA methylation program in developing hippocampus and its alteration by alcohol. Plos One 8, e60503.

Coppedè, F., Migliore, L., 2010. Evidence linking genetics, environment, and epigenetics to impaired DNA repair in Alzheimer's disease. J. Alzheimers Dis. 20, 953-966.

De Jager, P.L. Srivastava, G., Lunnon, K., Burgess, J. Schalkwyk, LC., Yu, L, Eaton, M.L. Keenan, B.T., Ernst, J., McCabe, C., Tang, A., Raj, T., Replogle, J., Brodeur, W., Gabriel, S., Chai, H.S., Younkin, C., Younkin, S.G., Zou, F., Szyf, M., Epstein, C.B., Schneider, J.A., Bernstein, B.E., Meissner, A., Ertekin-Taner, N., Chibnik, L.B., Kellis, M., Mill, J., Bennett, D.A., 2014. Alzheimer's disease: early alterations in brain DNA methylation at ANK1, BIN1, RHBDF2 and other loci. Nat. Neurosci. 17, 1156-1163.

Douaud, G., Refsum, H., de Jager, C.A., Jacoby, R., Nichols, T.E., Smith, S.M., Smith, A.D., 2013. Preventing Alzheimer's disease-related gray matter atrophy by B-vitamin treatment. Proc. Natl. Acad. Sci. U. S. A. 110, 9523-9528.

Falls, J.G., Pulford, D.J., Wylie, A.A., Jirtle, R.L., 1999. Genomic imprinting: implications for human disease. Am. J. Pathol. 154, 635-647.

Fuso, A., 2013. The 'golden age' of DNA methylation in neurodegenerative diseases. Clin. Chem. Lab. Med. 51, 523-534.

Fuso, A., Seminara, L., Cavallaro, R.A., D'Anselmi, F., Scarpa, S., 2005. S-adenosylmethionine/ homocysteine cycle alterations modify DNA methylation status with consequent deregulation of PS1 and BACE and beta-amyloid production. Mol. Cell. Neurosci. 28, 195-204.

Fuso, A., Nicolia, V., Ricceri, L., Cavallaro, R.A., Isopi, E., Mangia, F., Fiorenza, M.T., Scarpa, S., 2012. S-adenosylmethionine reduces the progress of the Alzheimer-like features induced by B-vitamin deficiency in mice. Neurobiol. Aging 33, e1-e16 (1482)

Gebel, T.W., 2002. Arsenic methylation is a process of detoxification through accelerated excretion. Int. J. Hyg. Environ. Health 205, 505-508.
Grimm, M.O., Rothhaar, T.L., Grosgenm, S., Burg, V.K., Hundsdorferm, B., Haupenthalm, V.J Friess, P., Kinsm, S., Grimm, H.S., Hartmann, T., 2012. Trans fatty acids enhance amyloidogenic processing of the Alzheimer amyloid precursor protein (APP). J. Nutr. Biochem. 23, 1214-1223.

Jang, H., Serra, C., 2014. Nutrition, epigenetics, and diseases. Clin. Nutr. Res. 3, 1-8.

Jiang, G., Xu, L., Song, S., Zhu, C., Wu, Q., Zhang, L., Wu, L., 2008. Effects of long-term low-dose cadmium exposure on genomic DNA methylation in human embryo lung fibroblast cells. Toxicology 244, 49-55.

Jin, P., Warren, S.T., 2000. Understanding the molecular basis of fragile X syndrome. Hum. Mol. Genet. 9, 901-908.

Jones, P.A., Buckley, J.D., 1990. The role of DNA methylation in cancer. Adv. Cancer Res. 54, $1-23$.

Kaliman, P., Párrizas, M., Lalanza, J.F., Camins, A., Escorihuela, R.M., Pallàs, M., 2011. Neurophysiological and epigenetic effects of physical exercise on the aging process. Ageing Res. Rev. 10, 475-486.

Kumar, A., 2000. Rett and ICF syndromes: methylation moves into medicine. J. Biosci. 25 213-214.

Li, Y.Y., Chen, T., Wan, Y., Xu, S.Q., 2012. Lead exposure in pheochromocytoma cells induces persistent changes in amyloid precursor protein gene methylation patterns. Environ. Toxicol. 27, 495-502.

Lu, S.C., 2000. S-adenosylmethionine. Int. J. Biochem. Cell Biol. 32, 391-395.

Lunnon, K., Smith, R., Hannon, E., De Jager, P.L., Srivastava, G., Volta, M., Troakes, C., Al-Sarraj, S., Burrage, J., Macdonald, R., Condliffe, D., Harries, L.W., Katsel, P., Haroutunian, V., Kaminsky, Z., Joachim, C., Powell, J., Lovestone, S., Bennett, D.A. Schalkwyk, L.C., Mill, J., 2014. Methylomic profiling implicates cortical deregulation of ANK1 in Alzheimer's disease. Nat. Neurosci. 17, 1164-1170.

Marques, S.C., Oliveira, C.R., Pereira, C.M., Outeiro, T.F., 2011. Epigenetics in neurodegeneration: a new layer of complexity. Prog. Neuropsychopharmacol. Biol. Psychiatry $35,348-355$.

Mastroeni, D., Grover, A., Delvaux, E., Whiteside, C., Coleman, P.D., Rogers, J., 2011. Epigenetic mechanisms in Alzheimer's disease. Neurobiol. Aging 32, 1161-1180.

McKay, J.A., Mathers, J.C., 2011. Diet induced epigenetic changes and their implications for health. Acta Physiol. 202, 103-118.

Morris, M.C., Evans, D.A., Bienias, J.L., Tangney, C.C., Hebert, L.E., Scherr, P.A., Schneider, J.A., 2005. Dietary folate and vitamin B12 intake and cognitive decline among communitydwelling older persons. Arch. Neurol. 62, 641-645.

Mukhopadhyay, P., Rezzoug, F., Kaikaus, J., Greene, R.M., Pisano, M.M., 2013. Alcohol modulates expression of DNA methyltransferases and methyl CpG-/CpG domain-binding proteins in murine embryonic fibroblasts. Reprod. Toxicol. 37, 40-48.

Olde Rikkert, M.G., Verhey, F.R., Sijben, J.W., Bouwman, F.H., Dautzenberg, P.L., Lansink, M. Sipers, W.M., van Asselt, D.Z., van Hees, A.M., Stevens, M., Vellas, B., Scheltens, P., 2014. Differences in nutritional status between very mild Alzheimer's disease patients and healthy controls. J. Alzheimers Dis. 41, 261-271.

Portela, A., Esteller, M., 2010. Epigenetic modifications and human disease. Nat. Biotechnol. 28, 1057-1068.

Roth, T.L., Sweatt, J.D., 2011. Epigenetic marking of the BDNF gene by early-life adverse experiences. Horm. Behav. 59, 315-320.

Shutoh, Y., Takeda, M., Ohtsuka, R., Haishima, A., Yamaguchi, S., Fujie, H., Komatsu, Y. Maita, K., Harada, T., 2009. Low dose effects of dichlorodiphenyltrichloroethane (DDT) on gene transcription and DNA methylation in the hypothalamus of young male rats: implication of hormesis-like effects. J. Toxicol. Sci. 34, 469-482.

Takiguchi, M., Achanzar, W.E., Qu, W., Li, G., Waalkes, M.P., 2003. Effects of cadmium on DNA-(Cytosine-5) methyltransferase activity and DNA methylation status during cadmium-induced cellular transformation. Exp. Cell Res. 286, 355-365.

Tsai, Y.T., Chang, C.M., Wang, J.Y., Hou, M.F., Wang, J.M., Shiurba, R., Chang, W.C., Chang, W.C., 2014. Function of DNA methyltransferase 3a in lead $\left(\mathrm{Pb}^{2+}\right)$-Induced Cyclooxygenase-2 gene. Environ. Toxicol. http://dx.doi.org/10.1002/tox.21976 (in press).

van Veldhoven, K., Rahman, S., Vineis, P., 2014. Epigenetics and epidemiology: models of study and examples. Cancer Treat. Res. 159, 241-255.

Wald, D.S., Kasturiratne, A. Simmonds, M., 2010. Effect of folic acid, with or without other B vitamins, on cognitive decline: meta-analysis of randomized trials. Am. J. Med. 123, 522-527.

Ye, J., Rensing, C., Rosen, B.P., Zhu, Y.G., 2012. Arsenic biomethylation by photosynthetic organisms. Trends Plant Sci. 17, 155-162.

Zhu, C.W., Sano, M., 2006. Economic considerations in the management of Alzheimer's disease. Clin. Interv. Aging 1,143-154. 